\title{
OTIMIZAÇÃO DO PROJETO DE TROCADORES DE CALOR DO TIPO AIR-COOLER PELO MÉTODO SIMULATED ANNEALING
}

\section{V. PASSOS, G.S. FERNANDES e A. L. H COSTA}

Universidade do Estado do Rio de Janeiro, Departamento de Operações e Projetos Industriais

E-mail para contato: lu_gdf@yahoo.com.br

RESUMO - Air-coolers são trocadores de calor baseados em uma corrente de ar que escoa externamente a um feixe de tubos aletados por meio de um sistema de ventilação. São muito empregados em unidades industriais localizadas onde a disponibilidade de água é limitada. Neste contexto, o presente estudo tem como objetivo a otimização do projeto destes equipamentos, visando reduzir seus custos. A função objetivo adotada contempla basicamente o investimento no resfriador, na bomba da corrente de processo, nas tubulações e válvulas juntamente com os custos operacionais relativos à energia requerida para o funcionamento da bomba e do sistema de ventilação. As restrições são representadas fundamentalmente por um limite mínimo de excesso de área que determina a viabilidade técnica do equipamento. Em função da natureza inteira das variáveis de projeto, a otimização é conduzida através do método meta-heurístico simulated annealing. Para um determinado serviço térmico, a rotina de otimização implementada no software Scilab 5.5.0 é capaz de localizar os parâmetros geométricos do equipamento que garantem sua viabilidade técnica e que estão associados a um menor custo financeiro.

\section{INTRODUÇÃO}

Air-coolers são trocadores de calor onde uma corrente que se deseja resfriar ou condensar escoa no interior de um feixe de tubos aletados e troca energia térmica com uma corrente de ar, oriunda de um ou mais ventiladores. O conceito de empregar o ar no resfriamento de correntes quentes em escala industrial surgiu por volta de 1920 no segmento de óleo e gás nos Estados Unidos.

Comparando um air-cooler com um trocador de calor do tipo casco-e-tubos que utiliza geralmente água como fluido frio, pode-se pontuar uma série de vantagens. A localização da planta onde opera um air-cooler não apresenta restrições, já que um equipamento que utiliza água necessita estar situado próximo à fonte. Quanto às incrustações que reduzem a eficiência do equipamento, o ar apresenta menor formação das mesmas.

No entanto, o ar é um fluido menos eficiente quanto à capacidade de trocar calor se comparado com a água. Dessa forma, a economicidade de um air cooler está diretamente ligada à identificação de um balanço adequado entre investimento e custos operacionais definidos durante o projeto de uma unidade de troca térmica. 
Apesar da sua importância e da sensibilidade do projeto no desempenho econômico do equipamento, um número relativamente pequeno de trabalhos abordou técnicas automáticas para o projeto deste tipo de equipamento (Conradie et al., 1998; González et al., 2001; Doodman et. al., 2009; Kashani et al., 2013; Manassaldi et al., 2014).

Neste contexto, o presente trabalho tem como objetivo a otimização do projeto de aircoolers, visando reduzir o custo total do projeto. A função objetivo adotada contempla os custos de investimento, manutenção e operação. Os custos de investimento e manutenção dizem respeito aos custos no resfriador, no sistema de ventilação, na bomba da corrente de processo, nas tubulações e válvulas e na estrutura do equipamento. Os custos operacionais são relativos à bomba e ao sistema de ventilação. As restrições são representadas fundamentalmente por um limite mínimo de excesso de área. O método selecionado para a otimização foi o método metaheurístico simulated annealing, ainda não explorado pela literatura para este fim.

\section{AVALIAÇÃO DO DESEMPENHO DO EQUIPAMENTO}

No contexto do procedimento de otimização, as rotinas de avaliação do trocador atuam em conjunto com o procedimento de otimização, de forma que cada cálculo da função objetivo corresponde à resolução das equações do modelo de forma a estabelecer o desempenho termofluidodinâmico do equipamento. De forma complementar, é também calculado o custo do equipamento, através de correlações econômicas apropriadas.

Com este intuito são apresentadas nesta seção as principais equações associadas à avaliação do desempenho da sequência de alternativas de projeto geradas ao longo da busca.

\subsection{Hipóteses e predeterminações}

Seguem as principais hipóteses e predeterminações utilizadas para o desenvolvimento do modelo matemático: (1) Propriedades físicas médias e constantes tanto da corrente de processo quanto para o ar; (2) Vazões e temperaturas constantes das correntes envolvidas; (3) Não há agentes naturais como radiação solar ou chuva/neve; (4) Arranjo triangular; (5) Fluido de processo escoa dentro dos tubos e o ar por fora.

\subsection{Equações Térmicas}

A área de troca térmica é calculada pela seguinte expressão:

$$
A_{r e q}=\frac{Q}{U \Delta T_{L M} F}
$$

onde $Q$ é a carga térmica do trocador, $A_{r e q}$ é a área requerida para o serviço, $\Delta T_{L M}$ é a média logarítmica da diferença de temperaturas entre as correntes e $F$ é o fator de correção. $\mathrm{O}$ coeficiente global de troca térmica $(U)$ é calculado por: 


$$
U=\frac{1}{\left(\left(\frac{1}{h_{i}}+R_{f i}\right) \frac{A_{t}}{A_{i}}+\left(\frac{\left.A_{t} \ln : \frac{D_{t e}}{D_{t i}}\right)}{2 \pi k_{t}}\right)+\frac{1}{n_{f} h_{a r}}+\frac{R_{a r}}{n_{f}}\right)}
$$

sendo $\mathrm{n}_{\mathrm{f}}$ a eficiência da superfície aletada, $\mathrm{h}_{\mathrm{i}}$ e $\mathrm{h}_{\mathrm{ar}}$ os coeficientes de convecção interno e do ar, $\mathrm{R}_{\mathrm{fi}}$ e $\mathrm{R}_{\mathrm{ar}}$ as resistências de depósito no interior e no exterior dos tubos, $\mathrm{D}_{\mathrm{te}} \mathrm{e} \mathrm{D}_{\mathrm{ti}}$ os diâmetros externo e interno e $\mathrm{k}_{\mathrm{t}}$ a condutividade dos tubos.

A área total externa dos tubos $\left(\mathrm{A}_{\mathrm{t}}\right)$, a área interna $\left(\mathrm{A}_{\mathrm{i}}\right)$ e a área externa $\left(A_{o}\right)$, por metro de tubo são calculados por:

$$
\begin{gathered}
A_{t}=2 N_{f} \frac{\pi}{4}\left(\mathrm{D}_{\mathrm{f}}^{2}-\mathrm{D}_{\mathrm{te}}^{2}\right)+N_{f} \pi D_{f} t_{f}+N_{f} \pi D_{t e} s_{f} \\
A_{i}=\pi D_{t i} \\
A_{o}=\pi D_{t e}
\end{gathered}
$$

onde $D_{f}$ é o diâmetro da aleta, $t_{f}$ é a espessura da aleta, $s_{f}$ é a distância entre as aletas e $N_{f}$ é o número de aletas por metro de tubo.

O coeficiente de convecção interno é calculado através de correlações dependentes do regime de escoamento. Para regime laminar, o coeficiente é calculado conforme a correlação de Sieder e Tate (Equação 6) ou correlação de Hausen (Equação 7) e para regime turbulento pela correlação de Gnielinsk (Equação 8).

$$
\begin{gathered}
h_{i}=\frac{k i}{D_{t i}} 1,86\left(\frac{R e P r}{L / D}\right)^{\frac{1}{3}} ; \operatorname{Re}<2300, \operatorname{Pr}<5 \\
h_{i}=\frac{k_{i}}{D_{t i}}\left(3,66+\left(\frac{0,0668 \frac{D}{L} \operatorname{Re} \operatorname{Pr}}{1+0,04\left(\frac{D}{L} \operatorname{RePr}\right)^{\frac{2}{3}}}\right)^{\frac{1}{3}}\right) ; \operatorname{Re}<2300 \operatorname{Pr}>5 \\
h_{i}=\frac{k_{i}}{D_{t i}}\left(\frac{\frac{f}{8}(\operatorname{Re}-1000) \operatorname{Pr}}{1+12,7\left(\frac{f}{8}\right)^{\frac{1}{2}}\left(\operatorname{Pr}^{\frac{2}{3}}-1\right)}\right) ; \operatorname{Re}>2300
\end{gathered}
$$

Da mesma maneira calcula-se o coeficiente de convecção externo aos tubos $\left(h_{o}\right)$ pelas Equações 9 a 12. Para tiragem induzida, são utilizadas as correlações de Briggs e Young, e Ward e Young (Equações 9 e 10) e para tiragem forçada as correlações de Gianolio e Cuti (Equações 11 a 12). 


$$
\begin{gathered}
h_{a r}=\frac{k_{a r}}{D_{t e}} 0,134 \operatorname{Re}_{a r}^{0,681} \operatorname{Pr}_{\mathrm{ar}}^{0,33}\left(\frac{\mathrm{s}_{\mathrm{f}}}{\mathrm{L}_{\mathrm{f}}}\right)^{0,2}\left(\frac{\mathrm{s}_{\mathrm{f}}}{\mathrm{t}_{\mathrm{f}}}\right)^{0,1134} ; N_{r}>6 \\
h_{a r}=\frac{k_{a r}}{D_{t e}} 0,134 \operatorname{Re}_{a r}^{0,681} \operatorname{Pr}_{a r}^{0,33}\left(\frac{s_{f}}{L_{f}}\right)^{0,2}\left(\frac{s_{f}}{t_{f}}\right)^{0,1134}\left(1+\frac{v_{a r}}{\left(N_{r}\right)^{2}}\right)^{-0,14} ; N_{r}<6 \\
h_{a r}=\frac{k_{a r}}{D t e} 0,271 \operatorname{Re}_{a r}^{0,685} \operatorname{Pr}_{a r}^{0,33}\left(\frac{A_{t}}{A_{o}}\right)^{-0,311} ; N_{r}>6 \\
h_{a r}=\frac{k_{a r}}{D_{t e}} 0,271 \operatorname{Re}_{a r}^{0,685} \operatorname{Pr}_{a r}^{0,33}\left(\frac{A_{t}}{A_{o}}\right)^{-0,311}\left(\frac{N_{r}}{6}\right)^{-0,138} ; N_{r}<6
\end{gathered}
$$

\subsection{Equações Hidráulicas}

O consumo de energia elétrica é diretamente ligado à queda de pressão. Dessa forma, para a avaliação do custo gerado por um air-cooler é necessário o cálculo da perda de carga no sistema de ventilação $\left(\Delta P_{s h}\right)$, sendo esta a soma da queda de pressão no feixe $\left(\Delta P_{b}\right)$ e da queda de pressão nos ventiladores e $\operatorname{plenum}\left(\Delta P_{p}\right)$ :

$$
\begin{gathered}
\Delta P_{s h}=\Delta P_{b}+\Delta P_{p} \\
\Delta P_{b}=\left(\frac{f_{1} N_{r} v_{v e n t}^{2}}{2}\right) \rho_{a r} \\
\Delta P_{p}=\frac{K v_{v e n t}^{2} \rho_{a r}}{2}
\end{gathered}
$$

A energia consumida pelo sistema de ventilação $\left(W_{m s}\right)$ é dada por:

$$
\begin{aligned}
& W_{f s}=\Delta P_{s h} \frac{\mu_{a r}}{\rho_{a r}} \\
& W_{m s}=\frac{\frac{W_{f s}}{E f}}{1000}
\end{aligned}
$$

Da mesma maneira, é calculado o consumo de energia do sistema de bombeamento hidráulico:

$$
\Delta P_{t}=\left(f \frac{N_{p t} L v_{t i}^{2}}{D_{t i} 2}+K N_{p t} \frac{v_{t i}^{2}}{2}\right)\left(\rho_{h}\right)
$$




\subsection{Equações Econômicas}

O custo total do equipamento é contabilizado por 3 componentes: o custo de investimento $\left(C_{c a p}\right)$, o custo de operação $\left(C_{o p}\right)$ e o custo com manutenção $\left(C_{\text {man }}\right)$ :

$$
C_{\text {total }}=C_{o p}+\text { FCR } C_{\text {cap }}+C_{\text {man }}
$$

onde FCR é um fator de custo fixo, que consiste em seguros, taxas, depreciação e etc. Correlações detalhadas para a determinação de cada termo da função custo podem ser encontradas em Conradie et al. (1998).

\section{MÉTODO SIMULATED ANNEALNG}

O método simulated annealing é um método de otimização meta-heurístico inspirado no processo de resfriamento de metais (Yang, 2010). Este método possui como característica a aceitação não só de alterações que melhoram a função objetivo, mas também que pioram, sendo aceitos de acordo com uma probabilidade descrita pelo método. Essa característica se apresenta como uma vantagem tendo em vista que evita a convergência em um mínimo local.

Na versão adotada no presente trabalho, o método é controlado fundamentalmente por dois parâmetros: a temperatura inicial $\left(T_{0}\right)$ e a taxa de resfriamento $(\alpha)$. A evolução da temperatura que controla o processo de aceite de novos pontos é calculada por:

$$
T(t)=T_{0} \alpha^{t}
$$

A probabilidade com que uma alteração na função objetivo seja aceita é calculada por:

$$
P=e^{-C_{t o t} / T}
$$

Cada ponto do espaço de busca, correspondendo a uma alternativa de projeto, é representado por um vetor dos diferentes parâmetros construtivos a serem otimizados. Desta forma, as transições entre diferentes pontos ao longo da busca (i.e. movimentos) são realizadas através do sorteio de um dos parâmetros seguida de um segundo sorteio que indica se a alteração em relação à configuração existente implicará em um aumento ou redução do valor atual.

$\mathrm{Na}$ formulação do procedimento de otimização, as variáveis foram: diâmetro externo dos tubos $\left(\mathrm{D}_{\mathrm{t}, \mathrm{e}}\right)$, razão de passo dos tubos $\left(\mathrm{L}_{\mathrm{p} t} / \mathrm{D}_{\mathrm{t}, \mathrm{e}}\right)$, número de passes nos tubos $\left(\mathrm{N}_{\mathrm{p}, \mathrm{t}}\right)$, comprimento dos tubos $(\mathrm{L})$, número de aletas por metro de tubo $\left(\mathrm{N}_{\mathrm{f}}\right)$, altura das aletas $\left(\mathrm{L}_{\mathrm{f}}\right)$, número de filas de tubos $\left(\mathrm{N}_{\mathrm{r}}\right)$, número de tubos por feixe $\left(\mathrm{N}_{\mathrm{t}, \mathrm{b}}\right)$, número de feixes por baia $\left(\mathrm{N}_{\mathrm{bbay}}\right)$, número de baias $\left(\mathrm{N}_{\text {bay }}\right)$, percentual de cobertura dos ventiladores $\left(\mathrm{A}_{\mathrm{fan}}\right)$ e temperatura de saída do $\operatorname{ar}\left(\mathrm{T}_{\mathrm{c}, \mathrm{o}}\right)$. 


\subsection{Restrições}

A restrição fundamental adotada corresponde à imposição de um excesso de área mínimo. Para garantir que na solução tal limite seja sempre respeitado, foi adotado um processo em duas etapas. Na primeira etapa, o procedimento de otimização é aplicado tomando como função objetivo a ser maximizada a diferença do excesso de área do trocador e o excesso de área mínimo. A solução deste problema corresponde à identificação de um trocador viável, sendo este o critério de parada da primeira etapa. Posteriormente na segunda etapa, partindo da solução viável encontrada na primeira etapa, o método simulated annealing é aplicado tomando como função objetivo a ser minimizada o custo. Nesta busca, alternativas de trocadores que não atendam a restrição são imediatamente descartadas, ou seja, o método segue uma trajetória onde apenas pontos viáveis são aceitos. $\mathrm{O}$ critério de parada adotado na segunda etapa interrompe a busca quando o algoritmo alcança um limite máximo de movimentos sem sucesso.

\section{RESULTADOS}

As rotinas de cálculo foram desenvolvidas no software Scilab 5.5.0 com o tempo de execução médio do programa em torno de 15 segundos. Com o intuito de testar a eficiência do método de otimização proposto, este foi aplicado para o serviço térmico caracterizado nas Tabelas 1 e 2 .

Tabela 1 - Dados do serviço térmico.

\begin{tabular}{|l|c|}
\hline Vazão Mássica Corrente de Processo(kg/s) & 69,44 \\
\hline Temperatura de Entrada $\left({ }^{\circ} \mathbf{C}\right)$ & 150 \\
\hline Temperatura de Saída $\left({ }^{\circ} \mathbf{C}\right)$ & 100 \\
\hline Temperatura de Entrada do $\mathbf{A r}\left({ }^{\circ} \mathbf{C}\right)$ & 25 \\
\hline
\end{tabular}

Tabela 2 - Parâmetros do problema.

\begin{tabular}{|l|c|l|l|c|}
\hline \multicolumn{2}{|c|}{ Parâmetros do Projeto } & \multicolumn{2}{c|}{ Propriedades Físicas } \\
\hline Tiragem & Forçada & Condutividade do tubo $(\mathrm{W} / \mathrm{m} . \mathrm{K})$ & 45 \\
\hline Horas de Operação & 8000 & Aceleração da gravidade $\left(\mathrm{m} / \mathrm{s}^{2}\right)$ & 9,81 \\
\hline Excesso de área mínimo & $20 \%$ & Massa específica da aleta $\left(\mathrm{kg} / \mathrm{m}^{3}\right)$ & 2700 \\
\hline Espessura dos tubos (m) & 0,0021 & Condutividade da aleta $(\mathrm{W} / \mathrm{m} . \mathrm{K})$ & 208 \\
\hline Comprimento da faixa de aletas por metro de tubo $(\mathrm{m})$ & 1 & Massa específica do tubo $\left(\mathrm{kg} / \mathrm{m}^{3}\right)$ & 7860 \\
\hline Diâmetro do motor do ventilador $(\mathrm{m})$ & 0,75 & Massa específica do fluido quente $\left(\mathrm{kg} / \mathrm{m}^{3}\right)$ & 800 \\
\hline Diâmetro da caixa do ventilador $(\mathrm{m})$ & 3 & Viscosidade do fluido quente $($ Pa.s) & 0,001 \\
\hline Eficiência do ventilador & 0,8 & Condutividade do fluido quente $(\mathrm{W} / \mathrm{m} . \mathrm{K})$ & 0,1211 \\
\hline Resistência de depósito externa & 0 & Capacidade calorífica do fluido quente $(\mathrm{J} / \mathrm{kg} . \mathrm{K})$ & 2093,4 \\
\hline Resistência de depósito interna & 0 & & \\
\hline
\end{tabular}




\section{CCoBege.}

19 a 22 de outubro de 2014

Florianópolis/SC

O domínio das variáveis que são otimizadas pelo método simulated annealing é listado simplificadamente na Tabela 3.

Tabela 3 - Domínio das variáveis otimizadas.

\begin{tabular}{|c|c|c|c|c|c|c|c|c|c|c|c|c|}
\hline $\mathbf{D}_{\mathbf{t e}}(\mathbf{m})$ & $\mathbf{L}_{\mathbf{p}} / \mathbf{D}_{\mathbf{t e}}$ & $\mathbf{N}_{\mathbf{p t}}$ & $\mathbf{L}(\mathbf{m})$ & $\mathbf{N}_{\mathbf{f}}$ & $\mathbf{L}_{\mathbf{f}}(\mathbf{m})$ & $\mathbf{t}_{\mathbf{f}}(\mathbf{m})$ & $\mathbf{N}_{\mathbf{r}}$ & $\mathbf{N}_{\mathbf{t b}}$ & $\mathbf{N}_{\text {bbay }}$ & $\mathbf{N}_{\text {bay }}$ & $\mathbf{A}_{\mathbf{f a n}}(\mathbf{\%})$ & $\mathbf{T}_{\mathbf{c o}}\left({ }^{\circ} \mathbf{C}\right)$ \\
\hline 0,00635 & 0,2 & 1 & 1,2 & 119 & 0,0047625 & 0,000381 & 1 & 190 & 1 & 1 & 40 & 30 \\
\hline 0,00953 & 0,21 & 2 & 1,8 & 158 & 0,0063500 & 0,000508 & 2 & 210 & 2 & 2 & 50 & 35 \\
\hline 0,0127 & 0,22 & & 2,4 & 197 & 0,0095250 & 0,000762 & 3 & 240 & 3 & 3 & 60 & 40,35 \\
\hline 0,01588 & 0,23 & & 3 & 237 & 0,0127000 & 0,000040 & 4 & 270 & 4 & & & 45,35 \\
\hline 0,01904 & 0,24 & & 3,6 & 276 & 0,0158750 & & 5 & 300 & 5 & & & 50,35 \\
\hline 0,0254 & 0,25 & & 4,2 & 315 & 0,0190500 & & 6 & 330 & 6 & & & 55,35 \\
\hline 0,03175 & & & $\ldots$ & 355 & 0,0254000 & & $\ldots$ & 390 & 7 & & & \\
\hline 0,0381 & & & 12 & 394 & & & 11 & & 8 & & & \\
\hline 0,04445 & & & 12,6 & 433 & & & 12 & & & & & \\
\hline 0,0508 & & & 13,2 & 473 & & & 13 & & & & & \\
\hline & & & 13,8 & 552 & & & 14 & & & & & \\
\hline & & & 14,4 & 630 & & & 15 & & & & & \\
\hline & & & 15 & 709 & & & 16 & & & & & \\
\hline
\end{tabular}

O programa foi executado três vezes consecutivas de forma independente e foram registrados na Tabela 4 os valores das variáveis otimizadas e a área em excesso.

Tabela 4 - Variáveis otimizadas.

\begin{tabular}{|c|c|c|c|c|c|c|}
\hline $\mathbf{D}_{\text {te }}(\mathbf{m})$ & $\mathbf{L}_{\mathbf{p t}}$ & $\mathbf{N}_{\mathbf{p t}}$ & $\mathbf{L}(\mathbf{m})$ & $\mathbf{N}_{\mathbf{f}}$ & $\mathbf{t}_{\mathbf{f}}(\mathbf{m})$ & $\mathbf{L}_{\mathbf{f}}(\mathbf{m})$ \\
\hline 0,03175 & 2,5 & 2 & 6 & 355 & 0,0003810 & 0,009525 \\
\hline 0,03175 & 2,5 & 2 & 6 & 355 & 0,0003810 & 0,009525 \\
\hline 0,0381 & 2,4 & 2 & 6,6 & 315 & 0,0005080 & 0,012700 \\
\hline $\mathbf{N}_{\text {tb }}$ & $\mathbf{N}_{\text {bbay }}$ & $\mathbf{N}_{\text {bay }}$ & $\mathbf{A}_{\text {fan }}(\boldsymbol{\%})$ & $\left.\mathbf{T}_{\mathbf{c o}}{ }^{\mathbf{}} \mathbf{C}\right)$ & $\mathbf{A}_{\text {excesso }}(\boldsymbol{\%})$ & $\mathbf{N}_{\mathbf{r}}$ \\
\hline 190 & 2 & 2 & 60 & 55,35 & 20,82873 & 3 \\
\hline 210 & 3 & 1 & 60 & 50,35 & 26,87226 & 3 \\
\hline 190 & 3 & 1 & 60 & 55,35 & 22,3678 & 3 \\
\hline
\end{tabular}

O custo associado a cada alternativa ótima está indicado na Tabela 5, onde é possível observar que apesar da natureza estocástica do método e da possibilidade de múltiplos ótimos locais, os trocadores ótimos encontrados possuem custos relativamente semelhantes. Visando mensurar o desempenho do método no sentido de redução de custos, na Tabela 5 também são apresentados o custo total do primeiro trocador viável encontrado, ou seja, o trocador que respeita o excesso mínimo de área correspondente a $20 \%$ em relação à área mínima requerida para realização do serviço térmico, obtido em cada caso. Partindo do primeiro trocador viável, os demais trocadores gerados foram apresentando valores de custo inferiores, sendo o trocador 
ótimo associado a, em média, a uma redução de custo de 59\%. Pode-se observar assim que o algoritmo levou a reduções de custo consideráveis ao final da busca.

Tabela 5 - Custo dos trocadores e redução de custo.

\begin{tabular}{|c|c|c|}
\hline Custo Total do Primeiro Viável Encontrado(\$) & Custo Total do Otimizado(\$) & Redução de Custo(\%) \\
\hline 59381,845 & 38955,359 & 34,40 \\
\hline 182923,71 & 40127,222 & 78,06 \\
\hline 108348,34 & 39341,347 & 63,69 \\
\hline
\end{tabular}

Média $=\mathbf{5 8 , 7 2 \%}$

\section{CONCLUSÃO}

Nesse estudo, foi desenvolvido e implementado um programa capaz de determinar o conjunto de parâmetros de um trocador de calor do tipo air-cooler associados a um custo mínimo de projeto para um dado serviço térmico.

O programa desenvolve de forma satisfatória e coerente cálculos relacionados à termofluidodinâmica do problema e aos custos envolvidos no projeto do equipamento. A metodologia de cálculo do custo abrange parcelas relacionadas ao investimento, manutenção e operação de forma detalhada.

A resolução do problema é feita pelo emprego do método de otimização meta-heurístico simulated annealing, que se mostrou eficiente na busca pelo ponto ótimo no exemplo de serviço térmico investigado. As soluções encontradas em corridas independentes foram relativamente semelhantes e indicaram reduções consideráveis do custo.

\section{REFERÊNCIAS BIBLIOGRÁFICAS}

CONRADIE, A.E.; BUYS, J.D.; KRÖEGER, D.G. Performance and Optimization of DryCooling System for Power Plants through SQP Methods. Applied Thermal Engin. v.18, p.25-45, 1998.

DOODMAN, A.R.; FESANGHARY, M.; HOSSEINI, R. A robust stochastic approach for design optimization of air cooled heat exchangers. App. Energy. v.86, p.1240-1245, 2009.

GONZÁLEZ, M.T.; PETRACCI, N.C; URBICAIN, M.J. Air-cooler heat exchanger design using successive quadratic programming SQP. Heat Transf. Eng. v.22, nº 33, 2001.

KASHANI, A.H.A.; MADDAHI, A.; HAJABDOLLAHI, H. Thermal-economic optimization of an air-cooled heat exchanger unit. App. Therm. Eng., v.54, p.43-55, 2013.

MANASSALDI, J.I.; SCENNA, N.J.; MUSSATI, S.F. Optimization mathematical model for the detailed design of air cooled heat exchangers. Energy, p.1-13, 2013.

YANG, X. S. Engineering optmization: an introducion with metaheuristic applications. Nova Jersey (EUA): Wiley, 2010. 